\title{
Size and plasticity effects in the compression zirconia micropillars
}

\author{
Erik Camposilvan ${ }^{1,2, *}$ and Marc Anglada ${ }^{1,2}$
}

${ }^{1}$ Department of Materials Science and Metallurgical Engineering, Universitat Politècnica de Catalunya, Av. Diagonal 647, 08028 Barcelona - Spain

${ }^{2}$ Center for Research in NanoEngineering CRnE, Universitat Politècnica de Catalunya, Av. Diagonal 647, Barcelona, 08028, Spain

* Corresponding author

E-mail: erik.camposilvan@upc.edu, erik.camposilvan@gmail.com

Phone number: +34934054452

Fax number: + 34934016706

\begin{abstract}
The micropillar compression technique has shown the potential for activating the brittle-to-ductile transition in ceramic monocrystals when testing reduced volumes. In this work, the role of size is studied by comparing the mechanical response of polycrystalline tetragonal zirconia micropillars and macroscopic specimens under compression. In micropillars, the absence of the natural defect population typical of bulk zirconia increases considerably the strength, allowing the activation of plastic deformation mechanisms and their study, showing in this way that the brittle-to-ductile transition is not limited to ceramic monocrystals only. The main mechanism of plastic deformation is transformation-induced plasticity, which is shown to be size dependent. The deformation behavior is studied in detail by loading-unloading tests at constant and increasing peak stresses, while the microstructure evolution is revealed by FIB cross-sections, TEM and STEM observations performed on lamellas extracted from pillars retrieved before failure. Finally, a failure mechanism is proposed, based on the damage induced by phase transformation.
\end{abstract}

Keywords: Zirconia, micro-pillar, phase transformation, transformation-induced plasticity, size effect 


\section{Introduction}

Intrinsic mechanical properties of materials, such as yield stress and strength, may exhibit an extrinsic behavior when their volume is greatly reduced. This tendency has been observed in monocrystals, where increasing yield stress is obtained by reducing the sample size. The existence of size effects has been highlighted by testing in different modes, such as micro- and nanoindentation [1,2], torsion tests [3], bending [4,5], tension [6] and compression [7].

The micropillar compression approach has received special attention due to several advantages. Firstly, the absence of important strain gradients that are usually responsible for substantial extrinsic contributions and can mask the effect of the sole volume and free surfaces [3,8]. Secondly, the relative simplicity of sample preparation and testing is another merit of micro-compression, which can be performed either ex-situ or insitu under SEM or TEM observation [9], as well as coupled with other electrical and acoustical [10], spectroscopic [11] and diffraction [12] techniques.

The limitations of this approach are mainly related to the micropillar taper angle, unavoidable in most of the sample preparation routes, and the confinement at the base of the specimens imposed by the surrounding material. Other limitations are related to the possible misalignment and the presence of friction forces between the micropillar top surface and the flat-punch compression tip. All these effects contribute to the nonuniformity of the micropillar stress state, making difficult to compare samples of different size and taper angle [13-15]. Misaligning of the system leads to underestimation of the elastic modulus, stress concentration at the pillar top edge, and the possibility of failure by buckling instead of compression [15]. Thermal and mechanical drift during testing may also be a source of errors in strain measurement [16].

By testing single-crystal micropillars of soft metals in compression one can study the activation of individual slip systems and the yield stress as a function of pillar size. An inverse power-law has been found between the critical resolved shear stress along the slip plane normalized by the shear modulus and the pillar diameter [14,17]. This relationship holds for FCC single crystals, for which the exponent is close to 0.6, while in BCC single crystals the behavior is somehow erratic and very sensitive to the initial dislocation density, with the yield stress also scaling with micro-pillar size trough an inverse power law, but now with lower exponents [14]. At the same time, both the extent of the size effect and the length scale where the transition 
between bulk and small-scale behavior takes place appear to depend on the bulk shear yield stress [18]. Thus, soft materials show more pronounced size effects and the transition occurs in bigger samples.

At present, full understanding of the behavior of polycrystalline micropillars is still lacking. In these materials a combination of extrinsic and intrinsic length scales seems to affect the overall behavior. Different and conflicting deviations from the Hall-Petch relation have been found by independent studies in polycrystalline wires, sheets and micro-pillars of FCC metals [19]. In particular, in nanocrystalline Nickel it has been shown that the "smaller being stronger" trend can be inverted depending on the ratio thickness/grain size [20]. As a result of dislocation dynamics simulations, two types of behavior have been deduced for metallic systems, depending on sample size, grain size, and dislocation density [21]. One is dominated by strain-hardening and follows the Hall-Petch relation; in the other type, plasticity is source-limited and the "smaller being stronger" behavior is expected, reflecting in part the tendency previously observed by Janssen et al. for Aluminum [22].

With reference to structural ceramics, testing of single-crystal micropillars has received much less attention. Nevertheless, this technique may be of particular interest to investigate the transition from brittle to ductile behavior in ceramics when the volume becomes small enough $[23,24]$. In this sense, Korte and Clegg [18] have shown that hard brittle materials like $\mathrm{MgO}$ can be deformed plastically at room temperature by micropillar compression. Once plasticity was activated, the size effect was similar to $\mathrm{BCC}$ metals for $\mathrm{MgO}$ soft slip systems, while hard slip systems showed a behavior rather similar to GaAs [25] and Si micropillars [26]. Deformation by dislocation glide during micropillar compression could also be activated at room temperature in high strength ceramic monocrystals like sapphire [27], silicon carbide [28] and silicon nitride [29]. With respect to zirconia, Lai and co-workers [30] have recently shown that single-crystal or oligocrystalline micropillars of zirconia highly doped with Ce and Y can display shape memory and superelastic effects in association with phase transformation under compression, while the presence of other plastic phenomena has not been discussed.

In the present work, the compression behavior of yttria-stabilized zirconia (3Y-TZP) micropillars has been studied and compared with the one of macroscopic samples. This material is composed of tetragonalmetastable polycrystals with $\sim 10$ vol.\% of stable cubic phase. It is well known that the transformation from the tetragonal phase into the stable monoclinic phase can be locally triggered, either mechanically by the 
presence of high stresses [31] or chemically by the diffusion of water species from the environment [31,32]. Here, 3Y-TZP micro- and nano-pillars of different sizes were milled by focused ion beam (FIB) and tested by monotonic and cyclic compression tests in order to study the role of size, phase transformation, plastic deformation and damage. All these aspects could be studied thanks to the considerable increase in strength of micropillars as compared to macroscopic specimens.

\section{Materials and Methods}

Commercial spray-dried zirconia powder (TZ-3YSB-E, Tosoh Corp.) was isostatically pressed at 200 MPa in a rod shape and sintered at $1450{ }^{\circ} \mathrm{C}$ in air inside a tubular furnace for 2 hours, obtaining a ceramic with density of $6.06 \pm 0.02 \mathrm{~g} / \mathrm{cm}^{3}(99.5 \pm 0.3 \%$ of the theoretical value) as measured by the Archimedes' method. The rod was cut into disks of approx. $1.5 \mathrm{~mm}$ thickness, which were ground and polished with diamond pastes down to less than $20 \mathrm{~nm} \mathrm{Ra}$. The disks were cut along the diameter and the resulting crosssection was polished using diamond films on a tripod fixture (Struers A/S) to reduce blunting of the crosssectional edge. The disk halves were mounted on inclinable SEM holders with hard conductive adhesive and coated with a thin carbon layer of $\sim 10 \mathrm{~nm}$. The holder was tilted conveniently to facilitate both the parallel and normal visualization of the sample surface by the electron and ion beams employed during micropillars milling in a Zeiss Neon 40 dual beam station. The surface was visualized tangentially by the ion beam to set the reference angle for milling and then the stage was tilted and orientated accordingly for starting the milling procedures. The micropillars were milled directly into the sample polished surfaces, close to the edge of the cross-section, in order to allow better visualization after testing. Milling was performed with a two-steps procedure that had been optimized for each pillar size in order to minimize the taper angle and maintain similar aspect ratios and reasonable milling times. In the first step a large well, $\sim 34 \mu \mathrm{m}$ in diameter, was attained with a relatively high ion current. The size of the well had been adapted to facilitate the compression experiments avoiding contact between the indenter tip and the surface and allowing to locate the pillar by the built-in optical microscopes mounted on the two nanoindenters employed. In the second step, lower currents were used and the milling profile, i.e. the dwell time associated to each pixel as a function of the distance from the pillar axis, was tuned for each pillar size. Four nominal sizes were selected: $3.3 \mu \mathrm{m}, 1 \mu \mathrm{m}, 0.65 \mu \mathrm{m}$ and $0.30 \mu \mathrm{m}$ in diameter. The aspect ratio was between 1:2 and 1:4 in all the pillars. A thin cyanoacrylate strip 
was manually deposited on the half disk from where the samples were milled, approximately $500 \mu \mathrm{m}$ away from the micropillars edge, serving as a soft surface for detecting the shape and location of the mark left by the flat-punch tip before testing. The two nanoindentation systems employed for testing were an MTS XP Nanoindenter (now Agilent, Oak Ridge, TN) and a CSM Ultra Nano Hardness Tester (CSM Instruments, Peseux, Switzerland). In both systems, the site of the indentation was first selected by imaging the surface with the optical microscope and then the indenter (or the sample, depending on the system) was moved to perform the indentation at the selected location. Therefore, the distance between the microscope and the indenter tip had to be properly calibrated. Prior to testing, the SEM holder was tilted to orientate the surface normally to the loading axis and mounted on supports designed for the indenter. Once inside the nanoindentation system, additional adjustments on the tilting angle were performed, if needed, by indenting the cyanoacrylate strip and visualizing the mark with the microscope until a regular shape was observed also at small indentation depths. The loading rates were adjusted to obtain similar strain rates $\left(1-3 \times 10^{-3} \mathrm{~s}^{-1}\right)$ for all the conditions and tests duration of approximately $40 \mathrm{~s}$ (monotonic tests to failure). Accurate microscope-toindenter calibrations were performed by indenting on the cyanoacrylate strip before each single test to guarantee the correct positioning of the flat-punch tip over the pillar head. This procedure was also useful for cleaning the indenter tip from residual debris from broken samples. Compression testing at constant loading rate was used in all the tests. Repeated loading/unloading tests until rupture were also performed by loading between a constant low load and either constant or increasing peak loads.

The pillars were visualized before and after testing. The actual diameter, height and taper angle of each pillar were measured from SEM micrographs. Since the maximum stress occurs at the pillar top, the initial diameter measured at this location was used in the calculation of stress. Load-displacement data were converted into stress-strain curves to compare different sample sizes. Some pillars were unloaded before breakage to observe their appearance by SEM and, in selected cases, to observe a FIB cross-section or to lift out a thin lamella from the pillar cross-section. The latter was observed first by Scanning Transmission Electron Microscopy (STEM) inside a Zeiss Neon 40 dual-beam station equipped with a STEM module at 30 $\mathrm{KV}$, obtaining bright field and annular dark field images. Conventional Transmission Electron Microscopy (TEM) observations were later performed with a JEOL $1200 \mathrm{EX}-\mathrm{II}$ at $120 \mathrm{KV}$. 
Macroscopic compression tests were carried out on 3Y-TZP prisms of 2 × 2 x $4 \mathrm{~mm}$ obtained by cutting and polishing the same sintered rods employed for milling micropillars. The prisms were tested in an Instron 8562 universal testing machine at strain rates similar as for the micropillars, transferring the load through two WC-Co plates and recording the displacement with an Instron LVDT 5809R transducer with +/- 1mm axial travel.

\section{Results}

Examples of stress/strain curves obtained during monotonic testing of micropillars are shown in Figure 1. The initial part of these curves shows increasing stiffness in association with the progressive contact between the indenter and the sample and the potential presence of debris/humidity on their surfaces. This is followed by a linear behavior, and finally a significant deviation from linearity with increasing curvature until failure. The compressive strength lies between 6.5 to $9.3 \mathrm{GPa}$, being much higher than for macroscopic specimens (3.5-4.3 GPa), which only show linear elastic behavior until rupture. Mean values of failure stress $\left(\sigma^{*}\right)$ and failure strain $\left(\varepsilon^{*}\right)$ obtained in monotonic tests of micropillars are reported in Table 1 , where $\varepsilon^{*}$ is the maximum strain measured at failure to which the initial strain associated to the contact has been subtracted.

Among the micropillars, no evident size effect in terms of strength is appreciated. In terms of strain, localized strain bursts are observed only for samples with diameter smaller than $1 \mu \mathrm{m}$, appearing sometimes at relatively low stress (e.g. 1700 MPa, see Fig. 1-D). Bigger micropillars show smooth curves, which are linear until $\sim 3 \mathrm{GPa}$, when they start to deviate. At low stresses and once the indenter-pillar contact is settled, the slope is between $3 \%$ to $37 \%$ smaller than the theoretical elastic modulus (210 GPa for $3 \mathrm{Y}-\mathrm{TZP}$ ). This variability is independent of the pillar size. On the other hand, when strain bursts appear in the $0.30 \mu \mathrm{m}$ micropillars, the slopes of the curve before and after the first discontinuous strain burst are similar.

The occurrence of transformation-induced plasticity was confirmed by SEM observation of micropillars tested to $85-90 \%$ of $\sigma^{*}$ and unloaded. The "stripes" observed on the pillar surface (Figures 2 and 3) are transformation bands, indicating extensive transformation in nearly all grains and suggesting a first explanation for the evident plasticity effects observed in the stress-strain curves. Irreversible deformation can be also appreciated in SEM pictures of the same micropillar (see Fig. 2, C-D and E-F and Fig. 3, A-B) by the 
permanent change in dimensions after unloading. The pillars appear slightly shorter and thicker once compressed.

Remarkable accumulation of martensitic plates was detected around the isolated alumina grains that are present in the microstructure as a result of the small amount of alumina in the composition of the starting powder (see Fig. 2-B).

Table 1. Comparison of failure stress $\left(\sigma^{*}\right)$ and failure strain $\left(\varepsilon^{*}\right)$ for micropillars and $2 \times 2 \times 4 \mathrm{~mm}$ bulk samples.

\begin{tabular}{|c|c|c|c|c|c|}
\hline & \multicolumn{4}{|c|}{ Micropillars } & \multirow{2}{*}{$\begin{array}{c}\text { Bulk } \\
\text { Samples } \\
\text { 2x2x4 mm }\end{array}$} \\
\hline & $3.3 \mu \mathrm{m}$ & $1 \mu \mathrm{m}$ & $0.65 \mu \mathrm{m}$ & $0.3 \mu \mathrm{m}$ & \\
\hline$\sigma^{*}(\mathrm{MPa})$ & $7442 \pm 788$ & $7061 \pm 563$ & $7658 \pm 523$ & $7489 \pm 912$ & $3907 \pm 291$ \\
\hline$\varepsilon^{*}(\%)$ & $6.85 \pm 1.23$ & $6.22 \pm 1.13$ & $6.88 \pm 0.85$ & $7.08 \pm 0.74$ & $\sim 2$ \\
\hline
\end{tabular}

The distribution of martensitic plates could be observed in greater detail from FIB cross-sections of tested micropillars (Fig. 3-D) and from the extracted lamellas observed by STEM (Fig. 4) and TEM (Fig. 5). It can be appreciated that the transformation is not limited to the surface but interests the whole volume, with higher concentration in the upper part of the pillar. In the STEM images, the martensitic plates associated with the formation of monoclinic phase are orientated mostly in the range $30^{\circ}-60^{\circ}$ with respect to the loading axis and spread in most of the grains. On the contrary, grains close (but external) to the base of the pillar are not transformed, indicating that sample preparation for electron microscopy did not introduce artifacts or relevant stresses in the foil. The presence of few isolated intergranular microcracks was sometimes detected on the surface or cross-sections of samples loaded up to $85-90 \%$ of the average strength and retrieved before failure. In the TEM micrograph of Fig. 5, four small grain-boundary microcracks can be appreciated, which were not observable in the STEM images. Small "wing cracks" could be sometimes observed developing at the tips of intergranular microcracks, like in the detail of Fig. 2-G. Moreover, TEM micrographs reveal the presence of some untransformed regions inside the micropillar. These regions have a distorted appearance, with the presence of strain-induced contrast and deformed Moiré fringes that may indicate the presence of dislocations. The distorted contour of some martensitic laths may also indicate other inelastic mechanisms activated after the transformation. 
SEM imaging of micropillars after failure shows mixed inter-trans-granular fracture, with the presence of many micro-cracks on the fracture surface as reported in Fig. 6-A, D, F. In some areas, the saw-toothed appearance reflects the contour of individual martensitic plates (Fig. 6-C). Extensive microcracking with evidence of crack merging appears on the external surface of broken micropillars (Fig. 6-B and E). The fracture plane is generally tilted with respect to the loading axis.

Examples of loading-unloading tests at increasing peak stress are presented in Fig. 7, where the irreversible component of the strain is clearly exhibited. For stresses higher than $3.5 \mathrm{GPa}$, immediate load reversal induced an unloading curvature at the peak load, which did not appear for load reversals at lower peak stresses. By holding the peak stress (hold time $=10 \mathrm{~s}$ ) instead of the immediate reversion, the deformation at constant peak stress increased with time and mostly during the first few hold seconds. This time-dependent strain at constant stress, which increases with the peak stress and only appears at stresses higher than $3.5 \mathrm{GPa}$ (see Fig. 7-B), should not be confused with the instantaneous strain bursts that were observed in sub-micron pillars loaded monotonically. In Fig. 7-B, it can be seen that during the unloading and reloading segments, a narrow hysteresis loop appears, whose width increases slightly with the peak stress. At peak stresses $\sim 3 \mathrm{GPa}$, the measured unloading elastic modulus was similar to the theoretical value of $210 \mathrm{GPa}$, while an increase of less than $20 \%$ was detected for higher peak stresses.

The response of $0.65 \mu \mathrm{m}$ micropillars to two cyclic loading-unloading tests at constant load amplitude is reported in Figure 7. The pillar was first cyclically loaded between $7.05 \mathrm{GPa}$ and $0.3 \mathrm{GPa}$ for 40 cycles and then the test was repeated by increasing the maximum stress to $8.40 \mathrm{GPa}$ until failure. At the lower stress amplitude, most of the plastic strain occurs in the first cycles, with little additional plastic strain in the subsequent cycles (see the insert of Figure 8). The unloading curvature at peak load is observed only during the first cycle. When the peak stress is increased to $8.4 \mathrm{GPa}$, the deformation behavior during the first two cycles evolves similarly as for the previous amplitude, but in the $3^{\text {rd }}$ cycle the loading-unloading loop starts to become wider, i.e. the cyclic plastic strain increases during the next cycles until failure occurs in the $6^{\text {th }}$ cycle. 


\section{Discussion}

The micropillars tested in this work range from the pure polycrystalline condition for $3.3 \mu \mathrm{m}$ pillars, where there are approximately 10 grains along the diameter, to the bamboo-like arrangement in the $\sim 0.3 \mu \mathrm{m}$ pillars, where there are only 0 to 2 grain boundaries along the diameter (oligo-crystalline pillars).

In the previous section, it has been shown that the compressive strength increases considerably for the micropillars (6.5-9.3 GPa) with respect to the macroscopic tests (3.5-4.3 GPa). This result can be partly related to the different nature of defects in macro- and micro- samples. Processing defects of a few tens of micrometers in size are present inside the macro-samples, as it can be calculated for the critical crack size from the strength of macroscopic flexure tests, which is typically of $\sim 1200 \mathrm{MPa}$. In fact, considering the value of 4.1 MPavm as $\mathrm{K}_{\mathrm{IC}}[33]$ and a semicircular defect with an appropriate geometric factor [34], the calculated radius is of $\sim 8 \mu \mathrm{m}$. On the other hand, small-scale samples are milled from hypothetically defect-free regions, and indeed no defect was observed by SEM on the surface and FIB cross-sections prior to testing, with the exception of nanopores (20-50 nm). The absence of preexisting flaws was also demonstrated for zirconia micro-beams tested in bending in Ref. [35].

Another important difference is suggested by the shape of stress-strain curves: linear elastic behavior until failure was observed in tests of macroscopic specimens, while significant deviation from the linear regime was recorded for the micropillars starting from $\sim 3 \mathrm{GPa}$ (for $3.3 \mu \mathrm{m}$ diameter micropillars), meaning that there is a transition from the brittle to quasi-plastic behavior between these length scales.

\subsection{Failure modes}

The generally accepted micromechanical model describing brittle failure in compression of macroscopic samples in absence of confinement is based on the existence of initial flaws or discontinuities. Under increasing loads, suitably oriented flaws with respect to the compression axis nucleate tension cracks (wing cracks) which grow and curve toward the compression direction until failure occurs by axial splitting [36] The failure mode tends to shift to faulting, or macroscopic shear failure, when lateral confinement is applied to the body in compression [37]. 
In our case, failure occurred along inclined planes, resembling the shear faulting mode. The fact that only few microcracks were detected on the surface, cross-sections or lamellas of retrieved micropillars after stressing up to $90 \%$ of the strength suggests that presumably most of the cracks are created at higher stresses close to the failure stress. In effect, a large number of microcracks were observed on the fracture and external surfaces of micropillars after failure (Fig. 6), showing evidence of interaction and merging of transformationinduced microcracks. The mixed fracture surface appearance is precisely the result of the combination of grain boundary crack formation (intergranular) and crack merging.

Indeed, transformation starts at much lower stresses without the simultaneous nucleation of a significant number of microcracks, as elucidated from both the microstructural observations and the deviation from linear behavior on the stress-strain curves. The hardening observed is likely to originate from the increasing stress necessary to induce transformation or other inelastic deformation mechanisms in residual tetragonal regions embedded in transformed monoclinic phase, in which the associated shape change may be hard to accommodate due to the constraints imposed by the surrounding transformed material and grain boundaries. While in monocrystals of tetragonal zirconia, like the ones tested by Lai and co-workers [30], martensitic transformation is simple, single variant and complete, in polycrystals the constraints associated with surrounding grains and triple points make it a more complex phenomenon. In this sense, Ueland et al. studied the cubic-monoclinic transformation of the shape-memory alloy $\mathrm{Cu}-\mathrm{Zn}$ - $\mathrm{Al}$ by tensile testing of microwires with different grain size, reporting that regions of the parent phase were left untransformed near grain boundaries, while near triple points the transformation was partial and multivariant. Finite-elements simulations showed that the stresses associated with transformation relax very rapidly away from these singular regions, which also act as preferential sites for the nucleation of flaws and limit the amount of strain that can be sustained $[38,39]$. The t-m transformation of zirconia under tensile stress was also modeled by Mamivand et al [40], who confirmed the localization of high stresses along triple points and grain boundaries using the phase-field method. Here, we found the presence of multivariant transformation that was not complete close to grain boundaries, with the development of cracks at the end of monoclinic laths (see arrows in Fig. 5), suggesting a similar mechanism.

The presence of high concentration of transformation bands around alumina grains observed in the micropillar of Fig. 2-B are likely to be the result of the stiffness mismatch between the two phases, which 
induces stresses at the interface during compression. In this case, the situation is similar but more severe than for grain boundaries and triple points, so the incompatible strain has to be accommodated by the formation of numerous multivariant martensitic laths, and flaws are likely to form at the interface when increasing the stress.

Following these concepts, it becomes clear that phase transformation occurs first in sites where the transformation strain is compatible with the microstructure constraints, while close to the failure stress it extends to regions where the incompatible strain and high local stresses associated to multivariant transformation are responsible for the simultaneous nucleation of many flaws that interact and merge leading to failure.

The size of the defect created with this damage process is in principle independent from the sample diameter, and it can be estimated to be smaller than one grain facet. Failure can occur when the density of microcracks is enough for merging, which may occur through the nucleation of wing cracks or by mode-II interaction, in view that both modes have been observed. The stress necessary for wing crack formation in brittle materials with the presence of small defects (typically several tens of micrometers) under compression can be estimated for macroscopic samples according to the model presented in Refs. [36,41]. Sub-micrometric micropillars, however, are initially free of such defects. The small cracks from which fracture is nucleated are rather formed as a result of plastic deformation at high stress.

\subsection{Transformation-induced plasticity}

The reduction of sample size and the absence of preexisting defects imply the transition to a quasi-plastic behavior in polycrystalline zirconia, showing strain hardening effects and irreversible deformation. The micropillars failure strains of $5.4 \%$ to $8.6 \%$ are considerably higher than for macroscopic samples, in which fracture occurs practically in the elastic regime at $\varepsilon^{*} \sim 2 \%$.

In nanometric micropillars, the bamboo-like structure allows transformation to begin earlier than in the micrometric ones, since grains are less constrained by the presence of none or only few grain boundaries and the shape change can be better accommodated by the free surfaces (for example, see Fig. 1-D). Strain bursts are in fact detected at stresses as low as, e.g. $1700 \mathrm{MPa}$ in these cases. While this is a continuous phenomenon in micrometric samples, it becomes a discrete phenomenon in sub-micrometric samples, where 
each event produces a relatively higher contribution to the total strain and between individual bursts the stressstrain behavior is linear. If we consider the minimum transformation stress of zirconia micropillars as the "critical shear stress", similarly as in single crystals that deform by slip, the rule "smaller being weaker" seems to apply here, meaning that the critical shear stress is smaller for smaller sizes. It is well known that constraints play an important factor in triggering the $\mathrm{t}-\mathrm{m}$ transformation as it can be seen from the change in the total free energy, $\Delta \mathrm{G}_{\mathrm{t}-\mathrm{m}}[42]$ :

$$
\Delta \mathrm{G}_{\mathrm{t}-\mathrm{m}}=\Delta \mathrm{G}_{\mathrm{C}}-\Delta \mathrm{U}_{\mathrm{SE}}-\Delta \mathrm{U}_{\mathrm{S}}
$$

$\Delta \mathrm{G}_{\mathrm{C}}\left(<0\right.$ when $\left.\mathrm{T}<\mathrm{M}_{\mathrm{S}}\right)$ is the chemical free energy change, which depends on temperature and composition, $\Delta \mathrm{U}_{\mathrm{SE}}(>0)$ is the strain energy change associated with the transformed region, which depends on the surrounding matrix and its stiffness, the size and shape of the region that transforms, and the presence of stresses, and $\Delta \mathrm{U}_{\mathrm{S}}(>0)$ is the change in energy associated with the creation of new interfaces. Since $\Delta \mathrm{U}_{\mathrm{SE}}$ depends on the volume surrounding the transformed region, when the diameter of the micropillar is similar to the grain size the increase in strain energy is reduced and the energetic barrier for $\mathrm{t}-\mathrm{m}$ transformation is overcome at lower stresses.

Therefore, the important parameter that determines the "smaller being weaker" behavior in our case is the enhanced surface/grain boundary ratio for specimens with small diameter and not the diameter itself. Due to the scatter of the yield stress in $0.3 \mu \mathrm{m}$ pillars, which must be orientation-related, a relation between yield stress and size cannot be established.

\subsection{Loading-unloading tests at increasing peak stress}

Loading-unloading tests at increasing peak stress show that the plastic component of the strain increases with the peak stress, indicating that a higher fraction of grains transforms at higher stresses and that the transformation under compression at these scales requires increasing mechanical work to proceed.

The slight variation of the unloading modulus with the peak stress could be related to the difference in the elastic modulus between tetragonal and monoclinic zirconia, being the second moderately higher $[43,44]$. Another explanation could be given by the change in diameter during testing due to the plasticity resulting from phase transformation and other possible inelastic phenomena, plus the volume increase associated to the 
transformation $(\sim 4.5 \%)$, which has to be accommodated in the radial direction. This effect is emphasized by the taper angle, as calculated by Zhang et al. [15]. On the contrary, cracks that may form or extend during loading do not reduce the stiffness of the pillars, confirming the results found by Subhash and Nemat-Nasser [45] during high strain rate loading of Y-TZP after complete transformation. In a parallel work, they excluded ferroelastic domain switching as a possible source of inelastic strain or stiffness variation during tesing [46].

The observed behavior is completely different for micropillars extracted from the monoclinic surface layer that forms on 3Y-TZP after hydrothermal degradation, which present extensive microcracking, as was reported in a previous work [47]. The slope of the linear part of loading curves in monoclinic degraded micropillars is less than half that of non-cracked tetragonal micropillars, due to the large population of orientated microcracks at the grain boundaries. Even though the unloading slope at the peak stress in loadingunloading tests of degraded micropillars also increased slightly with the peak stress, the slope change was associated to the variation in load-transfer area due to the closure of increasing fractions of cracks under the applied stress [47].

The unloading curvature at the peak stress, as well as the time-dependent deformation during the hold segment at the peak stress outlined in Fig. 7, represent anelastic effects that were not expected if phase transformation would be the sole plastic deformation mechanism. In fact, since the $\mathrm{t}-\mathrm{m}$ transformation does not need diffusion, it is an essentially instantaneous phenomenon. Although in nanoindentation experiments the presence of thermal drift may give a spurious strain contribution at constant load if not properly corrected, this possibility was carefully analyzed and discarded since in our case the time-dependent strains do not occur at stresses below $\sim 0.5 \sigma^{*}$ independently on the absolute value of the load and pillar size. Moreover, the same behavior was found when testing with different nanoindenters, that is, the total strain at constant stress increased with the peak stress, while the strain rate decreased with time, in contrast to thermal drift effects.

One may then speculate that dislocation glide could be the deformation mechanism responsible for the additional time-dependent strains. This is active in presence of high stresses and low thermal activation, occurring usually when $\sigma / \mathrm{G}>10^{-2}$. The activation of dislocation movement at room temperature does not normally occur in bulk ceramic specimens without confinement. This is no longer valid at the small scale, where, for example, monocrystals of alumina, chromia, silicon carbide and silicon nitride [27-29,48] have been deformed plastically under compression by slip. The participation of dislocation glide is likely to happen 
here since the stress level reached without fracture is comparable to that in confined deformation by grinding, where dislocation activity is dominant [49]. If we assume that in hardness testing the confinement allows plastic deformation to occur with a yield stress $\left(\sigma_{y}\right)$ of about $1 / 3$ of the macroscopic hardness value $(H)$ [50], it means that the threshold for plastic deformation in the present micropillars would be $\sim 4.2 \mathrm{GPa}$, value which is well below the observed compressive strength. However, since the ratio $\sigma_{\mathrm{y}} / \mathrm{H}=1 / 3$ may be higher for slip in hard materials [51,52], no definitive conclusion can be drawn from this argument. As a matter of fact, when performing nanoindentation experiments in 3Y-TZP, no evidence of slip lines are observed around the indent while they are clearly revealed in cubic zirconia, which has about $12 \%$ higher hardness [53]. Indeed, dislocation activity in 3Y-TZP is revealed by TEM observations of the region under Vickers macro-indents, showing the presence of a large hemispherical transformed zone containing an inner region with high dislocation density [54]. Thus, although the beginning of inelastic deformation is controlled by $\mathrm{t}-\mathrm{m}$ transformation, glide at higher stresses cannot be dismissed. After the sudden change to zero stress rate, the plastic deformation at high stresses and constant load could be therefore explained by the transient dislocation movement occurring before they are stopped during the hold segment.

The local shape change of the top region of some pillar (see e.g. Fig. 2-F) evidences the necessary existence of a plastic mechanism other than phase transformation, which could not produce such localized strain. In TEM images, both the presence of heavily distorted Moiré patterns and the blurred contrast exhibited by some distorted regions could indicate dislocation-rich areas, while individual dislocations are not resolved. These regions coincide mostly with the grains -or grain fractions- that did not undergo phase transformation and could not sustain the deformation by means of transformation-induced plasticity, although the presence of bended martensitic plates indicates that some transformed regions have been successively plastically deformed as well, similarly to what observed in the indentation plastic zone [54]. High resolution TEM studies are needed to clarify the presence and role of dislocations, which will be the subject of future studies.

\subsection{Cyclic loading-unloading tests at constant stress amplitude}

While cyclic hardening occurs during the initial cycles for the two cyclic peak stresses studied ( $7 \mathrm{GPa}$ and $~ 8.4 \mathrm{GPa}$ ), softening sets in after few cycles at the higher stress amplitude, leading rapidly to fracture. 
The widening of the loading loop just before fracture may be associated to the increase in the number and size of microcracks, which open and close during each compression cycle at a stress level close to the monotonic strength.

\subsection{Possible sources of artifacts}

\subsubsection{Effect of fib ion implantation}

The mechanical properties could be affected by Ga bombardment during pillar milling, as shown by Kiener et al. [55]. Several defects may appear, like point defects, dislocations and precipitates. The presence of these defects and the thickness of the affected layer depend on the energy of the ions and the impact angle. An amorphous layer forms at the surface, which may range from few $\mathrm{nm}$ in thickness to $\sim 50 \mathrm{~nm}$. The ion currents employed in our study suggest that our amorphous layer might be of not more than 10-20 nm thick, which was confirmed by TEM observations (see Fig. 5). The influence of this layer on dislocation movement in monocrystals might be important through the formation of dislocation pile-ups and the action of a back stress. In our case, where dislocation movement is not the main deformation mechanism, the amorphous layer is considered of minor importance. The nucleation of transformation occurs from internal stresses, so the surface modification induced by FIB may not alter this process.

\subsubsection{Taper effect and correction for surface deflection}

$1^{\mathrm{o}}$ to $3.5^{\mathrm{o}}$ of taper angle were measured on the micropillars of this study. The impossibility of reproducing exactly the same taper angle and aspect ratio for all the samples is a further explanation for the slight differences in the observed elastic modulus, failure stress and strain. The asymmetry in loading induced by taper angle introduces an additional driving force for axial crack propagation, as outlined in Ref. [56,57], which was estimated to be in the range 6-22\% for similar angles in Si micropillars. Since failure occurred by shear faulting rather than pure axial splitting, this contribution is considered of secondary importance. The elastic modulus measured from the stress-strain curves is affected by three main sources of error. First, since the pillar top was chosen for calculation, this leads to an overestimation of the elastic modulus. Other authors have chosen to use mean or equivalent diameters, which makes sense when energetic analysis are of interest but not in our case, where the failure stress is of more importance [30]. Second, the deflection of the surface at 
the base of the pillar has not been taken into account. This spurious strain component can be corrected according to the Sneddon model [58], as it was done in the work of Frick et al. [59]. For the maximum loads employed at the small scale in this study, the calculated correction is less than $10 \%$ for all micropillars. Due to the limited effect of this correction and the presence of other sources of error, data are presented without it. Third, the occurrence of buckling would result in lower elastic modulus and failure stresses. In the few cases where bucking was suspected, like in the pillar of Fig. 2-F, results were not reported.

Another point worthwhile mentioning is that the degree of phase transformation in the pillars is not homogeneous since the upper part in contact with the flat nanoindenter is more transformed than the bottom close to the base. This may be attributed to the smaller cross-section close to the top as well as to the effect of non uniform stresses near the contact and the presence of confinement in the lower part, which is likely to hinder the transformation.

\section{Conclusions:}

- In polycrystalline zirconia micropillars the absence of natural defects, typically found in macroscopic specimens, increases considerably the compressive strength up to $\sim 8 \mathrm{GPa}$. Thanks to the high stresses, plastic deformation mechanisms are activated, producing evident shape change and failure strains of $\sim 7 \%$, four times larger than in macroscopic tests. While the yield stress for plastic deformation is on average $\sim 3$ GPa in micropillars, strain bursts are detected well below this value when reducing their diameter.

- Transformation-induced plasticity is the main observed plastic deformation mechanism, which occurs without significant damage up to stresses of about $90 \%$ of the strength. The surface/grain boundary ratio is the parameter controlling the stress necessary for its activation.

- Additional strains set in right after dropping the stress rate to zero during a monotonic test, indicating a strain relaxation effect that may be associated to dislocation glide. The latter is assumed to play a role in the plastic deformation of micropillars, opening the path to further research.

- Cyclic loading-unloading tests on micropillars show the existence of cyclic hardening during the first cycles and softening close to fracture. 


\section{Acknowledgements}

The authors would like to acknowledge Prof. K. Leifer for the interesting discussions about electron microscopy images and for his support during the stay at Uppsala University. Prof. U. Wiklund and Dr. J. Heinrichs are kindly acknowledged for their assistance with the nanoindenter set-up, and O. Torrents is acknowledged for providing the in-situ video.

This work was supported by Ministry of Economy and Competitiveness (MINECO) under the project ref. MAT2011-23913 and by Government of Catalonia under the project ref. 2014SGR0137. Erik Camposilvan acknowledges Universitat Politècnica de Catalunya for the grant FPU-UPC.

\section{References}

[1] W.D. Nix, H. Gao, Indentation size effects in crystalline materials: A law for strain gradient plasticity, J. Mech. Phys. Solids. 46 (1998) 411-425.

[2] W.D. Nix, J.R. Greer, G. Feng, E.T. Lilleodden, Deformation at the nanometer and micrometer length scales: Effects of strain gradients and dislocation starvation, Thin Solid Films. 515 (2007) 3152-3157.

[3] N.A. Fleck, G.M. Muller, M.F. Ashby, J.W. Hutchinson, Strain gradient plasticity: theory and experiment, Acta Metall. Mater. 42 (1994) 475-487.

[4] T. Namazu, Y. Isono, T. Tanaka, Evaluation of size effect on mechanical properties of single crystal silicon by nanoscale bending test using AFM, J. Microelectromechanical Syst. 9 (2000) 450-459.

[5] C. Motz, T. Schöberl, R. Pippan, Mechanical properties of micro-sized copper bending beams machined by the focused ion beam technique, Acta Mater. 53 (2005) 4269-4279.

[6] D. Kiener, W. Grosinger, G. Dehm, R. Pippan, A further step towards an understanding of size-dependent crystal plasticity: In situ tension experiments of miniaturized single-crystal copper samples, Acta Mater. 56 (2008) 580 592.

[7] M.D. Uchic, D.M. Dimiduk, J.N. Florando, W.D. Nix, Exploring specimen size effects in plastic deformation of Ni3(Al, Ta), MRS Proc. 753 (2002) BB1.4.

[8] J.R. Greer, W.C. Oliver, W.D. Nix, Size dependence of mechanical properties of gold at the micron scale in the absence of strain gradients, Acta Mater. 53 (2005) 1821-1830.

[9] M. Legros, D.S. Gianola, C. Motz, Quantitative In Situ Mechanical Testing in Electron Microscopes, Mater. Res. Soc. Bull. 35 (2010) 354-360.

[10] A.B. Mann, D. van Heerden, J.B. Pethica, T.P. Weihs, Size-dependent Phase Transformations During Point Loading of Silicon, J. Mater. Res. 15 (2000) 1754-1758.

[11] K. Wasmer, T. Wermelinger, a. Bidiville, R. Spolenak, J. Michler, In situ compression tests on micron-sized 
silicon pillars by Raman microscopy—Stress measurements and deformation analysis, J. Mater. Res. 23 (2008) 3040-3047.

[12] H. Van Swygenhoven, S. Van Petegem, The use of Laue microdiffraction to study small-scale plasticity, Jom J. Miner. Met. Mater. Soc. 62 (2010) 36-43.

[13] D. Kiener, C. Motz, G. Dehm, Micro-compression testing: A critical discussion of experimental constraints, Mater. Sci. Eng. A. 505 (2009) 79-87.

[14] M.D. Uchic, P.A. Shade, D.M. Dimiduk, Plasticity of Micrometer-Scale Single Crystals in Compression, Annu. Rev. Mater. Res. 39 (2009) 361-386.

[15] H. Zhang, B.E. Schuster, Q. Wei, K.T. Ramesh, The design of accurate micro-compression experiments, Scr. Mater. 54 (2006) 181-186.

[16] W.C. Oliver, G.M. Pharr, An improved technique for determining hardness and elastic modulus using load and displacement sensing indentation experiments, J. Mater. Res. 7 (1992) 1564-1583.

[17] R. Dou, B. Derby, A universal scaling law for the strength of metal micropillars and nanowires, Scr. Mater. 61 (2009) 524-527.

[18] S. Korte, W.J. Clegg, Discussion of the dependence of the effect of size on the yield stress in hard materials studied by microcompression of MgO, Philos. Mag. 91 (2011) 1150-1162.

[19] M.G.D. Geers, W.A.M. Brekelmans, P.J.M. Janssen, Size effects in miniaturized polycrystalline FCC samples: Strengthening versus weakening, Int. J. Solids Struct. 43 (2006) 7304-7321.

[20] J.R. Greer, J.T.M. De Hosson, Plasticity in small-sized metallic systems: Intrinsic versus extrinsic size effect, Prog. Mater. Sci. 56 (2011) 654-724.

[21] P.S.S. Leung, A.H.W. Ngan, Size effect on the strength of micron-sized polycrystals - A dislocation dynamics simulation study, Scr. Mater. 69 (2013) 235-238.

[22] P.J.M. Janssen, T.H. de Keijser, M.G.D. Geers, An experimental assessment of grain size effects in the uniaxial straining of thin Al sheet with a few grains across the thickness, Mater. Sci. Eng. A. 419 (2006) 238-248.

[23] K. Kendall, The impossibility of comminuting small particles by compression, Nature. 272 (1978) 710-711.

[24] B.L. Karihaloo, The impossibility of comminuting small particles by compression, Nature. 279 (1979) 169-170.

[25] F. Östlund, P.R. Howie, R. Ghisleni, K. Leifer, W.J. Clegg, J. Michler, Ductile - brittle transition in micropillar compression of GaAs at room temperature, Philos. Mag. 91 (2011) 1190-1199.

[26] B. Moser, K. Wasmer, L. Barbieri, J. Michler, Strength and fracture of Si micropillars : A new scanning electron microscopy-based micro-compression test, (2007) 1-8.

[27] A. Montagne, S. Pathak, X. Maeder, J. Michler, Plasticity and fracture of sapphire at room temperature: Load- 
controlled microcompression of four different orientations, Ceram. Int. 40 (2014) 2083-2090.

[28] S. Kiani, K.W.K. Leung, V. Radmilovic, A.M. Minor, J.-M. Yang, D.H. Warner, et al., Dislocation glidecontrolled room-temperature plasticity in 6H-SiC single crystals, Acta Mater. 80 (2014) 400-406.

[29] T. Csanádi, N.Q. Chinh, P. Szommer, J. Dusza, Z. Lenčés, P. Šajgalík, Deformation and Fracture of $\beta$-Silicon Nitride Micropillars, J. Am. Ceram. Soc. Rapid Comm (2014) 1-4.

[30] A. Lai, Z. Du, C.L. Gan, C.A. Schuh, Shape memory and superelastic ceramics at small scales., Science. 341 (2013) 1505-8.

[31] R.C. Garvie, R.H.J. Hannink, R.T. Pascoe, Ceramic steel?, Nature. 258 (1975) 703-704.

[32] K. Kobayashi, H. Kuwajima, T. Masaki, Phase change and mechanical properties of ZrO2-Y2O3 solid electrolyte after ageing, Solid State Ionics. 3-4 (1981) 489 - 493.

[33] M. Turon-Vinas, M. Anglada, Fracture toughness of zirconia from a shallow notch produced by ultra-short pulsed laser ablation, J. Eur. Ceram. Soc. 34 (2014) 3865-3870.

[34] S. Strobl, P. Supancic, T. Lube, R. Danzer, Surface crack in tension or in bending - A reassessment of the Newman and Raju formula in respect to fracture toughness measurements in brittle materials, J. Eur. Ceram. Soc. 32 (2012) 1491-1501.

[35] E. Camposilvan, O. Torrents, M. Anglada, Small-scale mechanical behavior of zirconia, Acta Mater. 80 (2014) 239-249.

[36] S. Nemat-Nasser, H. Horii, Compression-induced nonplanar crack extension with application to splitting, exfoliation, and rockburst, J. Geophys. Res. 87 (1982) 6805.

[37] H. Horii, S. Nemat-Nasser, Brittle failure in compression: splitting, faulting and brittle-ductile transition, Philos. Trans. R. Soc. London. 319 (1986) 337-374.

[38] S.M. Ueland, Y. Chen, C.A. Schuh, Oligocrystalline Shape Memory Alloys, Adv. Funct. Mater. 22 (2012) $2094-$ 2099.

[39] S.M. Ueland, C.A. Schuh, Grain boundary and triple junction constraints during martensitic transformation in shape memory alloys, J. Appl. Phys. 114 (2013) 053503.

[40] M. Mamivand, M. Asle Zaeem, H. El Kadiri, Shape Memory Effect and Pseudoelasticity Behavior in Tetragonal Zirconia Polycrystals: A Phase Field Study, Int. J. Plast. 60 (2014) 71-86.

[41] M.F. Ashby, S.D. Hallam, The failure of brittle solids containing small cracks under compressive stress states, Acta Metall. 34 (1986) 497-510.

[42] F.F. Lange, Transformation toughening - Part 1 Size effects associated with the thermodynamics of constrained transformations, J. Mater. Sci. 17 (1982) 225-234. 
[43] E.Y. Fogaing, Y. Lorgouilloux, M. Huger, C.P. Gault, Young's modulus of zirconia at high temperature, J. Mater. Sci. 41 (2006) 7663-7666.

[44] X.S. Zhao, S.L. Shang, Z.K. Liu, J.Y. Shen, Elastic properties of cubic, tetragonal and monoclinic ZrO2 from first-principles calculations, J. Nucl. Mater. 415 (2011) 13-17.

[45] G. Subhash, S. Nemat-Nasser, Uniaxial stress behaviour of Y-TZP, J. Mater. Sci. 28 (1993) 5949-5952.

[46] G. Subhash, S. Nemat-Nasser, Dynamic Stress-Induced Transformation and Texture Formation in Uniaxial Compression of Zirconia Ceramics, J. Am. Ceram. Soc. 76 (1993) 153-165.

[47] E. Camposilvan, M. Anglada, Micropillar compression inside zirconia degraded layer, J. Eur. Ceram. Soc. (2015).

[48] D.D. Stauffer, Deformation Mechanisms in Nanoscale Brittle Materials, Univerity of Minnesota, 2011.

[49] J.A. Muñoz Tabares, E. Jiménez-Piqué, J. Reyes-Gasga, M. Anglada, Microstructural changes in 3Y-TZP induced by scratching and indentation, J. Eur. Ceram. Soc. 32 (2012) 3919-3927.

[50] D. Tabor, The hardness of metals, Clarendon Press, Oxford, UK, 1951.

[51] J.J. Gilman, Relationship Between Impact Yield Stress and Indentation Hardness., J. Appl. Phys. 46 (1975) 1435-1436.

[52] L.J. Vandeperre, W.J. Clegg, The correlation between hardness and yield strength of hard materials, in: Mater. Sci. Forum, Trans Tech Publications, 2005: pp. 555-560.

[53] Y. Gaillard, M. Anglada, E. Jiménez-Piqué, Nanoindentation of yttria-doped zirconia: Effect of crystallographic structure on deformation mechanisms, J. Mater. Res. 24 (2009) 719-727.

[54] A. Pajares, F. Guiberteau, K.H. Westmacott, A. Dominguez-Rodriguez, TEM characterization of indented polycrystalline Y-PSZ, J. Mater. Sci. 28 (1993) 6709-6714.

[55] D. Kiener, C. Motz, M. Rester, M. Jenko, G. Dehm, FIB damage of Cu and possible consequences for miniaturized mechanical tests, Mater. Sci. Eng. A. 459 (2007) 262-272.

[56] P.R. Howie, S. Korte, W.J. Clegg, Fracture modes in micropillar compression of brittle crystals, J. Mater. Res. 27 (2011) 141-151.

[57] K. Kendall, Complexities of Compression Failure, Proc. R. Soc. London A. 361 (1978) 245-263.

[58] I.N. Sneddon, The relation between load and penetration in the axisimmetric boussinesq problem for a punch of arbitrary profile, Int. J. Eng. Sci. 3 (1965) 47-57.

[59] C.P. Frick, B.G. Clark, S. Orso, a. S. Schneider, E. Arzt, Size effect on strength and strain hardening of smallscale [ $\left.\begin{array}{lll}1 & 1 & 1\end{array}\right]$ nickel compression pillars, Mater. Sci. Eng. A. 489 (2008) 319-329. 
Fig. 1. Stress-strain response of micropillars of different sizes $(0.3 \mu \mathrm{m}-3.3 \mu \mathrm{m})$. Failure is marked with a star.

Fig. 2. A-B: Evidence of transformation on the surface of a $0.65 \mu \mathrm{m}$ diameter pillar after testing to $6 \mathrm{GPa}$ (unloaded before failure). Martensitic laths seem to concentrate around the visible alumina grain. C-D: Shape change in a $0.65 \mu \mathrm{m}$ pillar after loading to $6.1 \mathrm{GPa}$. E-F: shape change in a $0.30 \mu \mathrm{m}$ pillar with evidence of plastic deformation at the top and buckling (loading curve not included in the results). G: surface of a $3.3 \mu \mathrm{m}$ pillar unloaded before failure. Arrows indicate the presence of microcracks.

Fig. 3. A-B: $3.3 \mu \mathrm{m}$ diameter micropillar before and after testing (loaded up to $6.3 \mathrm{GPa}$ ), highlighting the change in size, side view. Vertical lines are due to curtain effect during the milling procedure. C: detail showing transformation on the surface and the presence of surface flaws, indicated by arrows. D: FIB crosssection of the same pillar, showing extensive phase transformation.

Fig. 4. A-B: $0.65 \mu \mathrm{m}$ diameter pillar before and after loading up to $6.1 \mathrm{GPa}$. C-D: Lamella preparation after Pt deposition and SEM observation of the prepared lamella. E: dark field STEM image of the lamella. F: bright field of the upper part of the pillar, showing the preferential inclination of transformation bands. G: Detail of the triple points close to the pillar top. The top-right edge of the pillar has been milled away during the depostition of the Pt protective layer.

Fig. 5. Bright field TEM images of the micropillar previously imaged by STEM in Fig. 4. The pillar is embedded in a nanocrystalline Pt layer needed for the lift-out procedure. Loading took place from the top-left corner in the axial direction. In A, arrows indicate visible microcracks associated to phase tranformation. In B, which is the bottom region of $\mathrm{A}$ at higher magnification, arrows indicate a region with distorted Moiré patterns.

Fig. 6. Appearance of four $3.3 \mu \mathrm{m}$ diameter pillars after failure. A and D: fracture surface with mixed intergranular-transgranular aspect plus the presence of growing intergranular microcracks. B and E: external surface of a second pillar, with evidence of crack merging and wing crack formation. $\mathrm{C}$ and F: details of the fracture surface from other two pillars, where the saw-toothed contour induced by transformation bands is observed in some areas.

Fig. 7. A: progressive loading of a $0.65 \mu \mathrm{m}$ diameter sample until 7.6 GPa, no hold. The detail shows the presence of a curvature in the upper part of the unloading curve. B: Progressive loading until rupture, $10 \mathrm{~s}$ hold segment at maximum load. The hysteresis loop in the unloading-reloading cycle is indicated. The insert shows the displacement at constant peak load.

Fig. 8. Cyclic loading tests of a $0.65 \mu \mathrm{m}$ diameter pillar. First test: 40 cycles, max load $7050 \mathrm{MPa}$. Second test: max load $8400 \mathrm{MPa}$, failure of the pillar at the $6^{\text {th }}$ cycle. 
Click here to download high resolution image
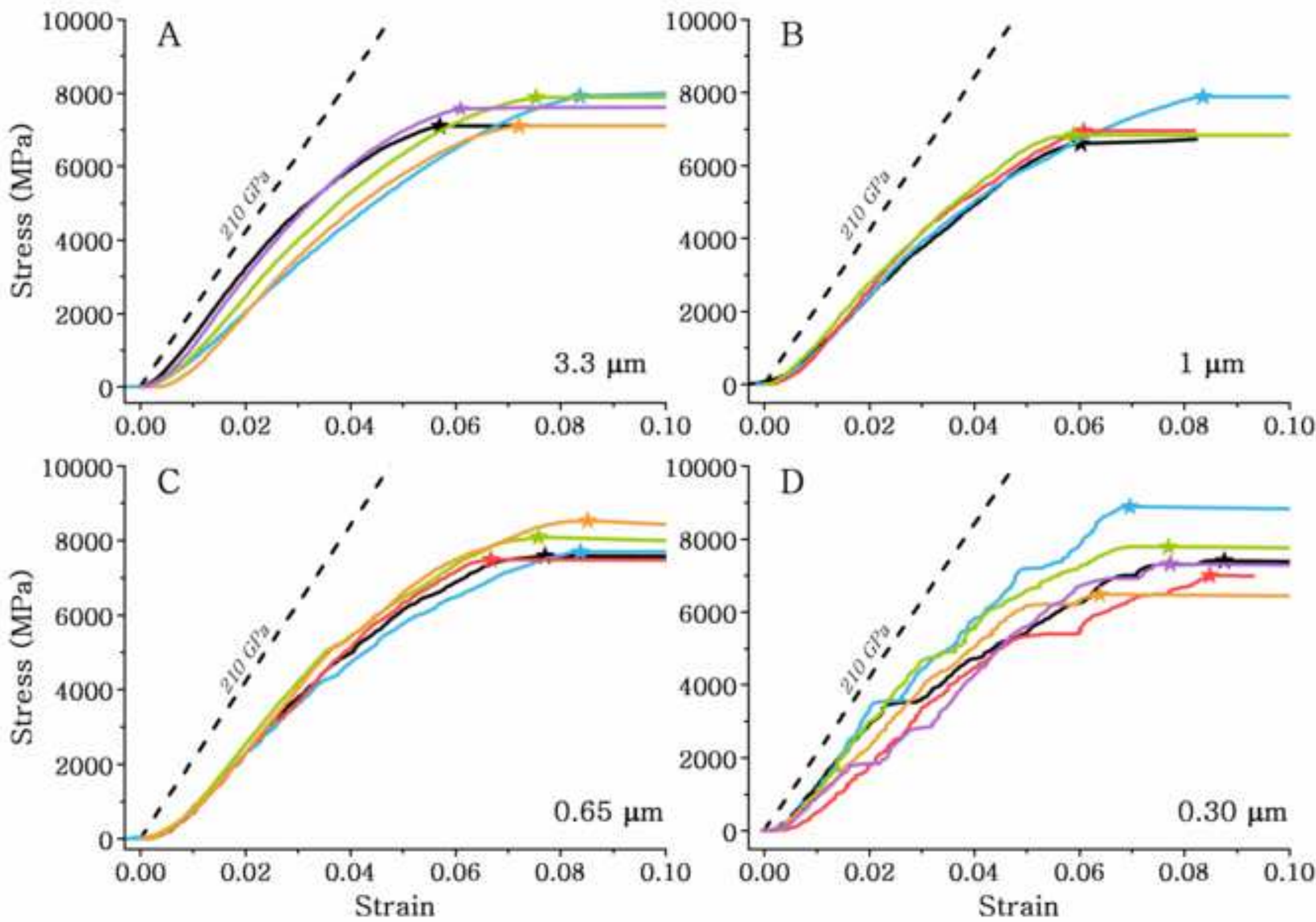


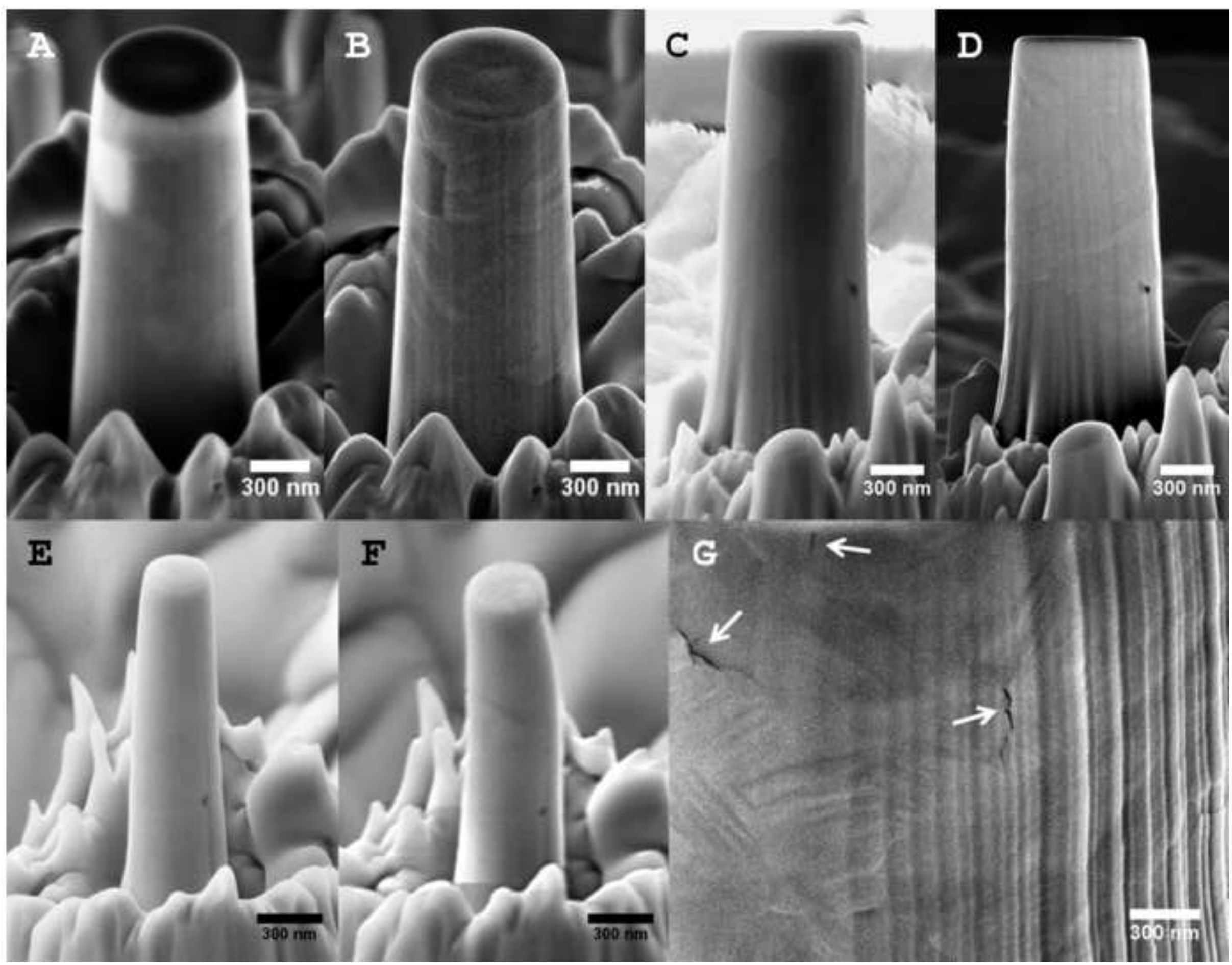


Click here to download high resolution image
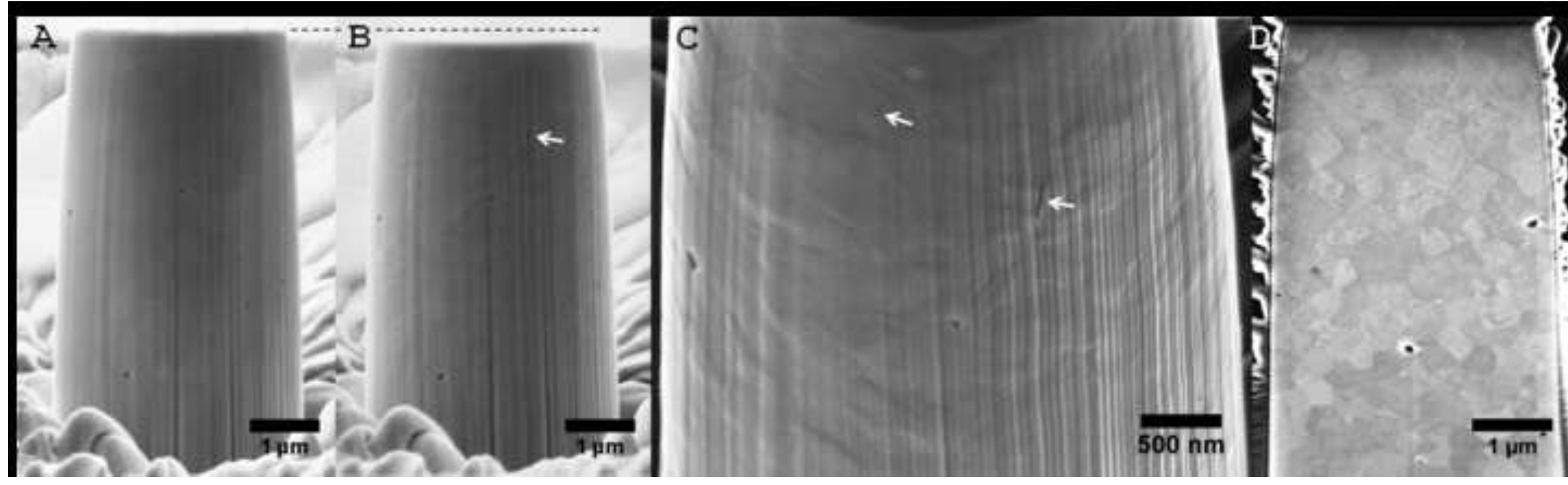
Figure 4
Click here to download high resolution image

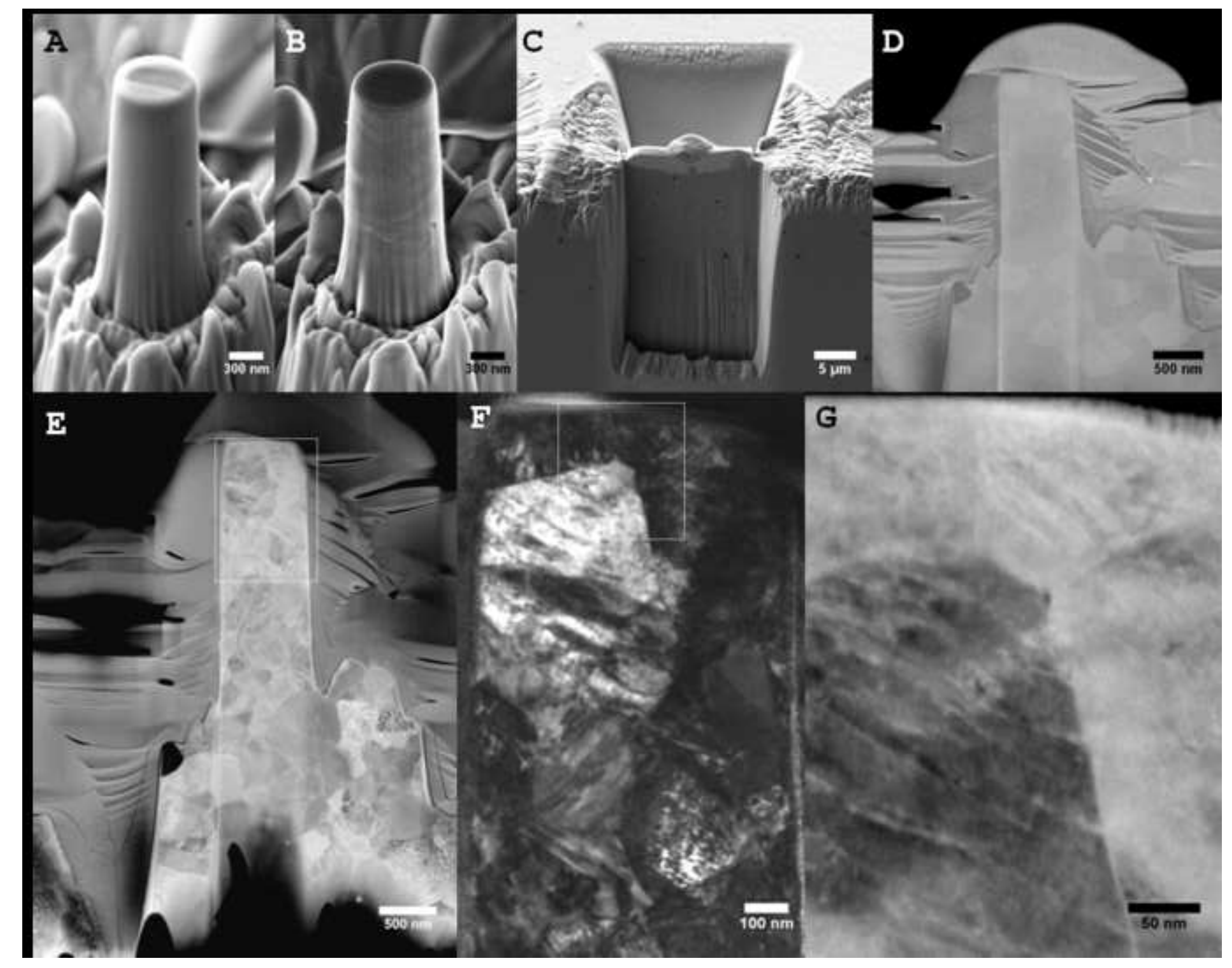




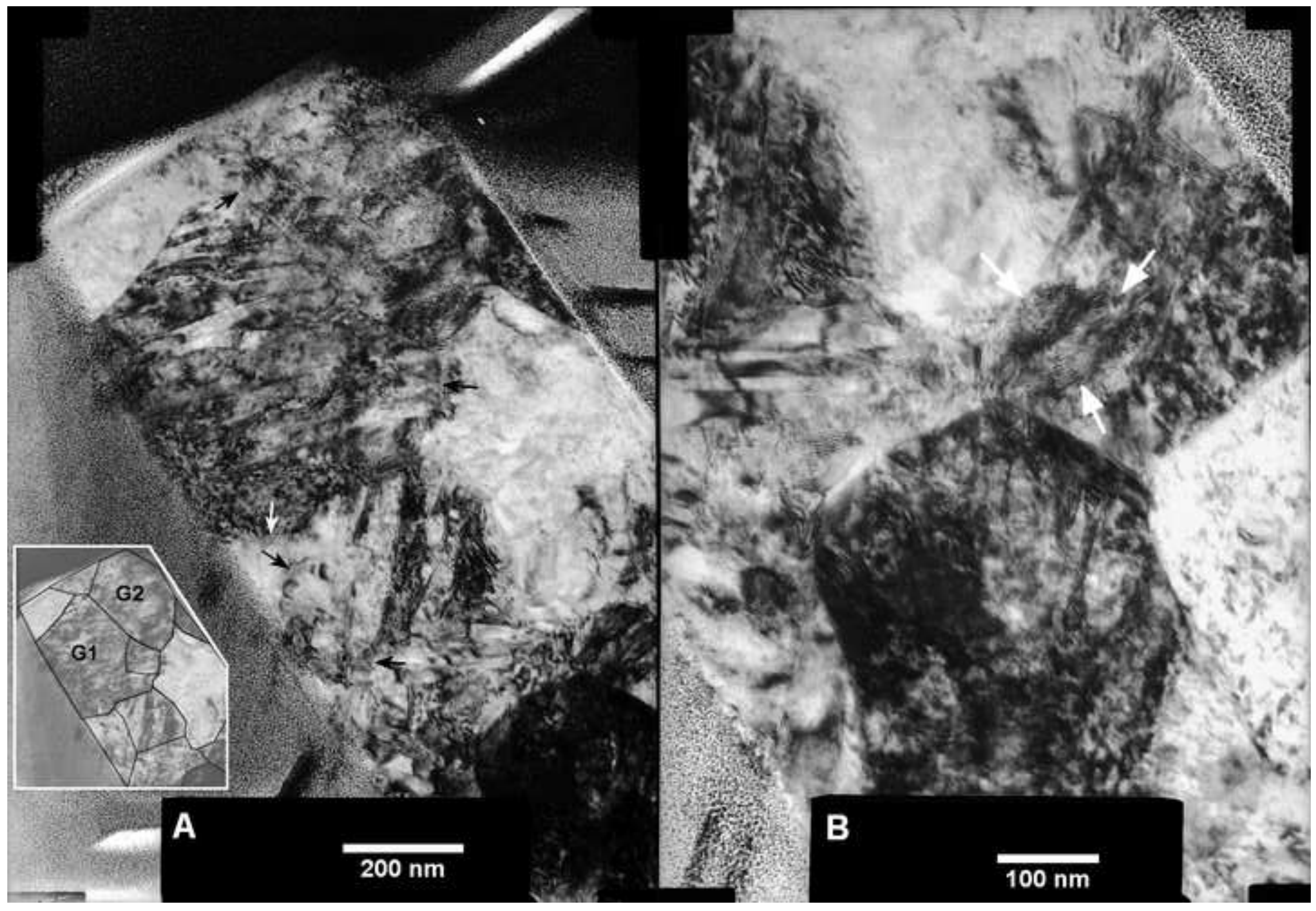


Click here to download high resolution image
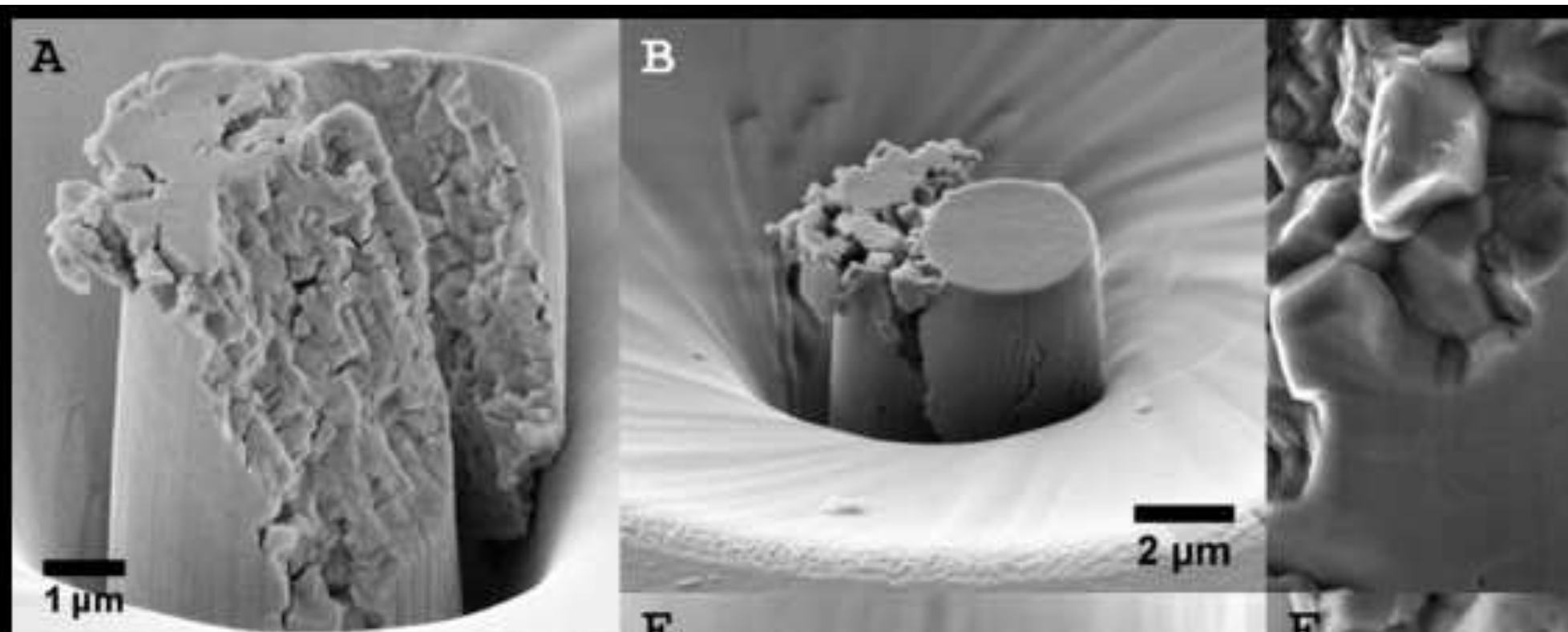

C
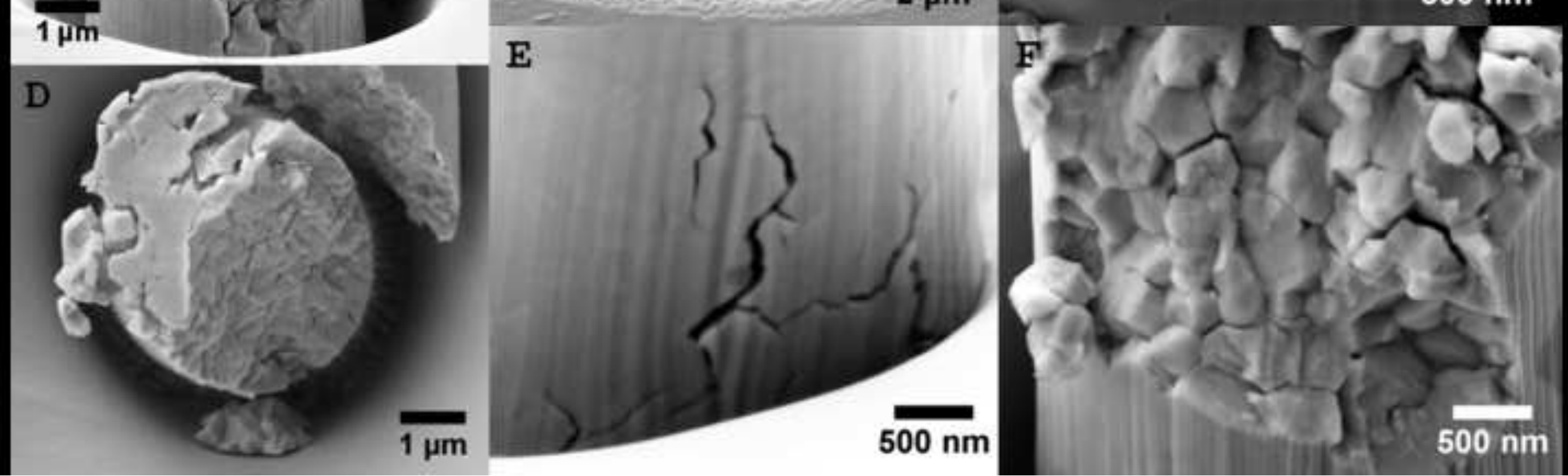

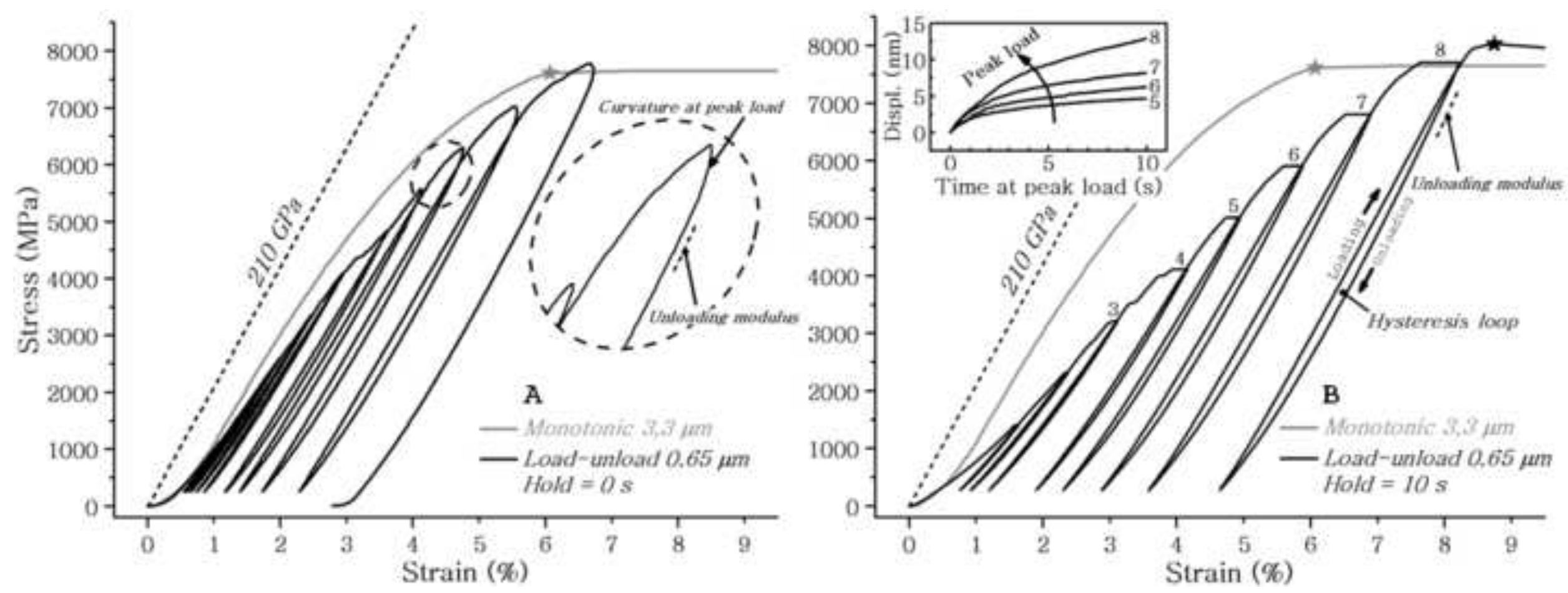


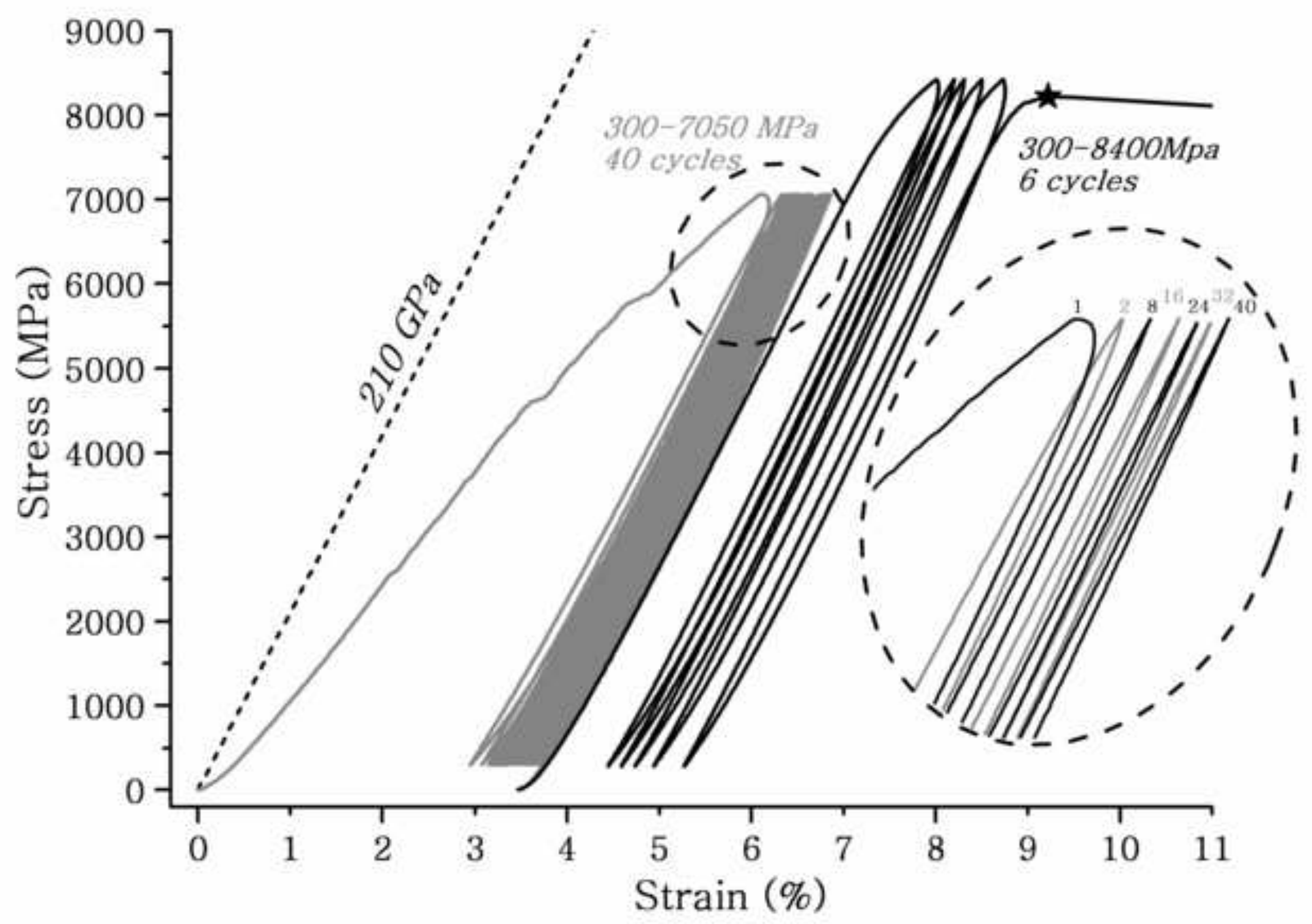

